\title{
Examination Regarding Change of Differences in the Diet Effect with Age: Analysis Based on Wavelet Interpolation Model
}

\author{
Katsunori Fujiii ${ }^{1, *}$, Tohru Ishigaki², Kohsuke Kasuya ${ }^{2}$ \\ ${ }^{1}$ Graduate School of Business Administration and Computer Science, Aichi Institute of Technology, Toyota City, Japan \\ ${ }^{2}$ Department of Exercise and Health Science, Aichi University of the Arts, Nagakute City, Japan \\ Email address: \\ fujii $a$ aitech.ac.jp (K. Fujii), isshy $a$ mail.aichi-fam-u.ac.jp (T. Ishigaki), kohsuke28kasuya $a$ gmail.com (K. kasuya) \\ ${ }^{*}$ Corresponding author
}

\section{To cite this article:}

Katsunori Fujii, Tohru Ishigaki, Kohsuke Kasuya. Examination Regarding Change of Differences in the Diet Effect with Age: Analysis Based on Wavelet Interpolation Model. American Journal of Sports Science. Vol. 8, No. 4, 2020, pp. 89-94. doi: 10.11648/j.ajss.20200804.12

Received: October 5, 2020; Accepted: October 20, 2020; Published: October 26, 2020

\begin{abstract}
During diets, people basically try to change their living habits to lose weight, such as restricting caloric intake and increasing energy consumption through exercise and activity. This could truly be considered a hostile act against corpulence in modern society. For many women in particular, the act of dieting is essentially wishful thinking in their daily lives, and selecting easy diet food would seem to be an obvious way to achieve this wish. However, while the diet effect must be clear when selecting better diet products, there have been no findings on diet effect that consider the effects of age. In this study, we used product evaluation, sex, age, height, body weight before use, body weight after use, duration of use, and other factors in data from the T. M. Community Co., Ltd. (TMC) review site "Diet Cafe," which specializes in diet products. We analyzed the changes in the diet effect with age by applying the wavelet interpolation model to the change in body weight and the percentage change in body weight in subjects aged from the $10 \mathrm{~s}$ to $60 \mathrm{~s}$. In addition, with the curves describing the amount and percentage of the change in body weight, we investigated the inflection point (critical point) in the diet effect with age by deriving velocity curves as differentials. The WIM was applied to the age-related changes in the size of the diet effect, and the identification of the age of 30 as a critical point in the diet effect from the behavior of the velocity curve may be considered a finding derived for the first time. The slowing of the velocity then becomes consistent about 10 years after the critical point, at around the age of 40 , from which it may be proposed that the age of 40 is a true transition phase for women.
\end{abstract}

Keywords: Diet Effect, Change with Age, Wavelet Interpolation Model, Critical Point

\section{Introduction}

One hypothesis on the origin of diets is that they began in the USA in 1880-1930, a time of change in how people were eating [1]. This was appropriately described in the book "Fat History" by Stearns [2] as the beginning of a war in which being overweight was the enemy in the middle class in the USA between 1890 and 1910, and which has continued to the present. From this description, the aversion to behaviors that do not get rid of excess weight serves to increase the value of the act of dieting in relative terms. The authors believe that diets are currently derived and are spreading around the world without relation to the concept of health. General diets adopt the method of changing living habits to lose weight, such as by limiting caloric intake and increasing the amount of energy consumed through exercise and activity. However, while short-term changes in living habits are thought to be possible, they are difficult to continue long-term. Because of this, interest is shown in the use of products that declare a weight-loss function or diet information with a low level of difficulty. With the growth of mass media in modern society, becoming the weight people see on television has permeated the popular mind. That is, modern values are that being thin is beautiful in terms of appearance. Therefore, many females have the desire to lose weight easily. This leads to increases in the size of the market for diet foods, which means increases in the number of people consuming health-related foods and the amount of money spent. A system of Foods 
with Functional Claims was begun in April 2015, and in the two years after it was started about $40 \%$ of foods delivered to the Consumer Affairs Agency were supplements, suggesting the possibility that supplements form the core of functional foods. Many of the foods called supplements are drink type or are provided in the form of tablets or capsules like medicine [3]. In a study of Japanese nationals aged from 40 to 82 years old, $55 \%$ of men and $61 \%$ of women reportedly used supplements [4]. There is no question that the expected effect of diet food and information is weight loss. In advertisements for the sale of such products, the weight loss effect is sometimes presented as the change in body weight before and after the use of those products. New diet products seem to come on the market every year recently, and much diet information can be found with the spread of the Internet. In these circumstances, information that suggests the efficacy of diet products has an important meaning. In fact, T. M. Community Co., Ltd, (TMC) has operated a website called "Diet Cafe" since 2008 that specializes in personal reviews of diet products. This website maintains a policy of neutrality; it does not sell any diet products or carry affiliate offers. As of August 2020, it had a total of more than 160,000 posts, and looking only at diet products it is one of the largest review sites in Japan. It also lists more than 8,000 diet products that are used in Japan. Thus, diet effects can be investigated with the use of this site. Previous research on the effects of diets $[5,6]$ has not gone beyond determining the weight change results from before to after the use of diet products [7]. Such methods have also been attempted in examining the effects of physical training, but in most of the protocols the effects of aging were not established because of the amount of data. Especially, while it is thought the influence of age cannot be ignored when investigating the effects of diets, currently there virtually no findings on the results of diets at different ages. This study used the diet review data set provided by TMC to the Research Organization of Information and Systems, and analyzed the changes with age in the effects of diets by applying the wavelet interpolation model to the amount of change in weight and the percentage change in weight in girls and women from the $10 \mathrm{~s}$ to $60 \mathrm{~s}$. We also investigated inflection points (critical points) with aging in the diet effects by deriving velocity curves as differentials. The meaning of those inflection points is then discussed.

\section{Methods}

\subsection{Materials}

The data used in the analysis were a review database from among the diet review data set provided by TMC to the Research Organization of Information and Systems. Since the content of the posts was not digitized, the text in the posts was excluded and the product ID, evaluation, sex, age, height, body weight before use, body weight after use, duration of use, and tags from that database were used. The time period recorded in the database was from July 2008 to June 2019.
The total number of data posts was 160,118 . The items assessed were the posters' subjective evaluation of the diet product, using a radio button format with "no rating" and ratings on 5 levels from 1 to 5 . Sex was recorded as female or male with a radio button format. Age was recorded with a pull-down menu as $<15$ years old, then in one-year increments from age 15 to 59 years old, and as $\geq 60$ years old for all ages beyond that. Height was recorded with a pull-down menu as $<140 \mathrm{~cm}$, then in $1 \mathrm{~cm}$ increments from $140 \mathrm{~cm}$ to $179 \mathrm{~cm}$, and as $\geq 180 \mathrm{~cm}$ for all heights beyond that. Body weight before use was the poster's body weight before the use of the product and, similarly, body weight after use was the poster's body weight after use of the product. Using a pull-down menu, body weight was recorded as $<30 \mathrm{~kg}$, then in $1 \mathrm{~kg}$ increments from $30 \mathrm{~kg}$ to $99 \mathrm{~kg}$, and as $\geq 100 \mathrm{~kg}$ for all weights beyond that.

\subsection{Data}

From all of the data (160,118 posts), posts in which sex was not recorded (77,905 posts) were excluded. There were then 6,878 posts from men and 75,335 posts from women. Since the amount of data for men was only about one-tenth that for women, only the data for women were used in the analysis. From that data, data for which the duration of use was "no use" were excluded. Data for which body weight before or after use of a product was $<30 \mathrm{~kg}$ or $\geq 100 \mathrm{~kg}$ were excluded. The change in body weight was then taken as the weight after use of the product subtracted from the body weight before use of the product. Similarly for height, data of $<140 \mathrm{~cm}$ and $\geq 180 \mathrm{~cm}$ were excluded, and BMI was calculated when body weight data were also obtained. With these procedures, the parameters of change in body weight, BMI before use, BMI after use, and change in BMI were added. The data used in the numerical analysis were height, body weight before use, body weight after use, change in body weight, BMI before use, BMI after use, and change in BMI. Based on the respective means and standard deviations, data outside the range of mean $\pm 3 \mathrm{SD}$ were rejected as outliers.

\subsection{Analysis}

The figure for the amount of change in body weight identified from the data was taken as the diet effect size. The figure obtained by dividing the amount of change in body weight by the body weight before use (change in body weight $\div$ pre-use body weight) was taken at the diet effect rate. Statistics by age were calculated in 10-year increments from the $10 \mathrm{~s}$ to the $60 \mathrm{~s}$ for the diet effect size (change in body weight). The statistics for 10 -year age cohorts were adapted to age axes of 15 years old, 25 years old, 35 years old, ... 65 years old. The diet effect size was inserted onto these age axes and least squares approximation regression polynomials were configured. Similarly, the least squares approximation regression polynomials were also applied to the diet effect rate. After grasping the trends, rigorous age-related change curves were analyzed with the wavelet interpolation model, and an evaluation chart was constructed. Velocity curves were also derived from differentials with the wavelet interpolation model, and inflection points with age were identified. 


\subsection{Analytical Methods}

The Wavelet Interpolation Model (WIM) interpolates between data points with a wavelet function and draws a growth distance curve to approximately describe the true growth curve from growth data. It is a method to differentiate the distance curve drawn in this way and derive the obtained growth velocity curves in order to investigate growth distance curves at the pubertal peak or the age of menarche. The WIM has been shown to sensitively read local phenomena and to have a very high approximation accuracy. Details of the theoretical background and the evidence for its effectiveness have been reported in previous studies [8-11], and so the data analysis method with the WIM will be omitted here. With regard to its application to the data on diet effect with age, the WIM was applied to the change in body weight (diet effect size) at age $15,25, \ldots 65$. The age axes of 10 -year age cohorts described in the previous section and the inflection points were then identified from the velocity curves derived from differentiation of the distance curves.

\section{Results}

\subsection{Changes with Age in Diet Effect Size (Change in Body Weight)}

Table 1 shows the means and standard deviations for the amount of change in body weight as the diet effect size for subjects from their $10 \mathrm{~s}$ to $60 \mathrm{~s}$. The mean change in body weight was about $1 \mathrm{~kg}$ for all ages, but there was huge variability. There was also a tendency for the amount of change in weight to gradually decrease with age. To elucidate this trend, the WIM was applied to the change in weight based on aging, as a result of which the change in weight showed a decreasing tendency while a linear change was shown with age (Figure 1). Moreover, a local peak phenomenon derived from the velocity curves was detected, and this peak could be identified as an inflection point for the diet effect size (change in weight) with age (Figure 2). The inflection point was at 30 years old. The age of 30 can be indicated as a critical point for differences in the diet effect. Phenomenologically for the diet effect, an effect is demonstrated from the age of about 40 , when the behavior of the velocity curve becomes gentler.

Table 1. Statistics of diet effect size.

\begin{tabular}{|c|c|c|c|c|c|c|c|c|}
\hline Age & Number & & $\begin{array}{l}\text { Height } \\
(\mathrm{cm})\end{array}$ & $\begin{array}{l}\text { Body Weight } \\
\text { before use (kg) }\end{array}$ & $\begin{array}{l}\text { Body Weight } \\
\text { after use (kg) }\end{array}$ & $\begin{array}{l}\text { Change in body } \\
\text { Weight (kg) }\end{array}$ & $\begin{array}{l}\text { Percentage change in } \\
\text { body Weight }(\%)\end{array}$ & Change in BMI \\
\hline \multirow{2}{*}{ All } & \multirow{2}{*}{48106} & Average & 158.38 & 58.04 & 57.20 & -0.84 & -0.01 & -0.33 \\
\hline & & $\mathrm{SD}$ & 5.12 & 8.21 & 8.16 & 1.92 & 0.03 & 0.77 \\
\hline \multirow{2}{*}{10 ’s } & \multirow{2}{*}{1742} & Average & 157.36 & 54.12 & 53.08 & -1.04 & -0.02 & -0.42 \\
\hline & & SD & 5.23 & 7.45 & 7.28 & 1.88 & 0.03 & 0.76 \\
\hline \multirow{2}{*}{ 20’s } & \multirow{2}{*}{11510} & Average & 158.26 & 56.16 & 55.05 & -1.11 & -0.02 & -0.44 \\
\hline & & SD & 5.22 & 7.90 & 7.77 & 1.93 & 0.03 & 0.78 \\
\hline \multirow{2}{*}{30 ’s } & \multirow{2}{*}{15725} & Average & 158.43 & 58.11 & 57.21 & -0.90 & -0.01 & -0.36 \\
\hline & & SD & 5.12 & 8.24 & 8.15 & 1.94 & 0.03 & 0.77 \\
\hline \multirow{2}{*}{ 40’s } & \multirow{2}{*}{14584} & Average & 158.67 & 59.42 & 58.77 & -0.65 & -0.01 & -0.26 \\
\hline & & $\mathrm{SD}$ & 5.01 & 8.07 & 8.04 & 1.88 & 0.03 & 0.75 \\
\hline 50 ’s & 4188 & Average & 158.01 & 59.82 & 59.30 & -0.52 & -0.01 & -0.21 \\
\hline \multirow{2}{*}{60 ’s } & \multirow{2}{*}{357} & Average & 157.13 & 59.92 & 59.46 & -0.45 & -0.01 & -0.18 \\
\hline & & $\mathrm{SD}$ & 5.35 & 8.25 & 8.15 & 1.99 & 0.03 & 0.81 \\
\hline
\end{tabular}

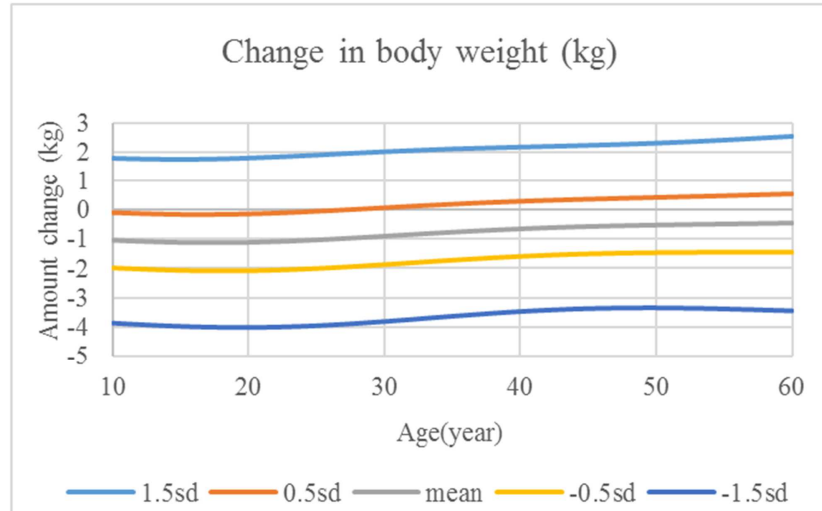

Figure 1. Age-related change evaluation chart of change in body weight.

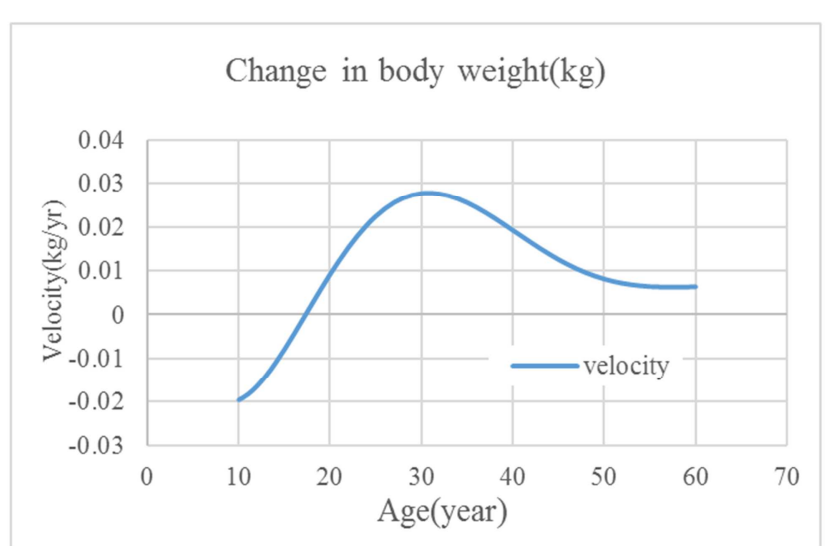

Figure 2. Velocity curve of change in body weight derived from wavelet interpolation model. 


\subsection{Changes with Age in the Diet Effect Rate (Percentage Change in Body Weight)}

Depending on the original body weight, the amount of change in weight as the diet effect size produces different proportional changes. Therefore, to maintain consistency in the amount of change, the percentage change in body weight was obtained by dividing the amount of change in body weight by the original weight. The rate of change in body weight was given consistency as the diet effect rate. Table 1 shows the age-related changes in the weight change rate. As in the previous section, the WIM was applied to the age-related change in the percentage change in body weight, and a trend similar to that for age-related change in body weight was shown (Figure 3). The inflection point from the velocity curve was at 30 years old, and the age of 30 can be indicated as a critical point for the difference in the diet effect rate (Figure $4)$.

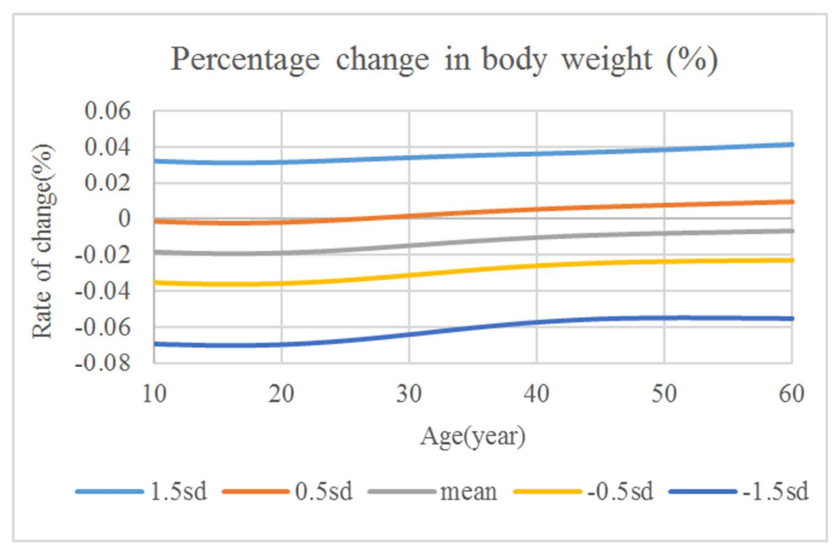

Figure 3. Age-related change evaluation chart of percentage change in body weight.

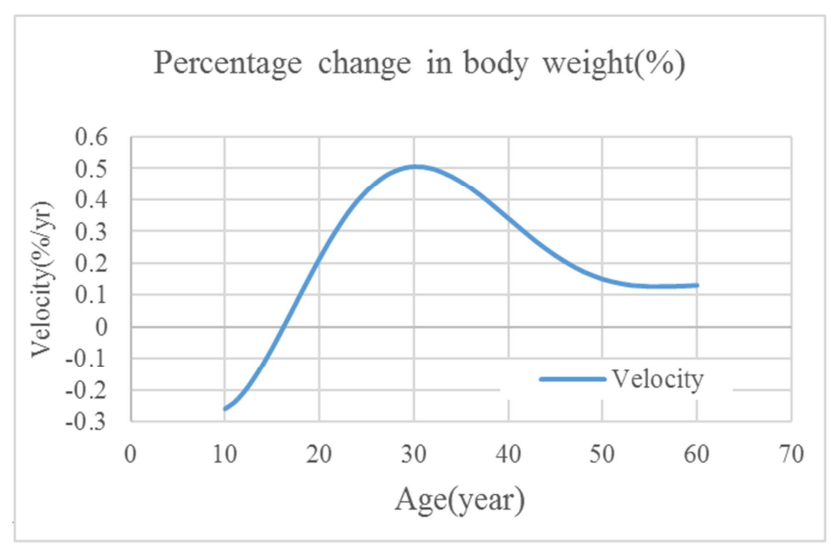

Figure 4. Velocity curve of percentage change in body weight derived from wavelet interpolation model.

\section{Discussion}

In dieting, there is an aversion to behaviors that do not eliminate excess weight and a relative heightening of the value on behaviors for the loss of weight. However, while diet behaviors are possible if short-term changes are sought, difficult elements increase when they become long-term and strategies that depend on simple methods are considered. For example, interest is shown in the use of products that promise weight-loss functions just by taking them and diet information with low levels of difficulty. Health-related products continued to grow in 2019 and the size of the market in fiscal 2019 was estimated at 1.45 trillion yen. Moreover, in fiscal 2019, the sixth year of the Foods with Functional Claims system, these products reached 260 billion yen on a manufacturer shipment base [12]. This growth in market size shows increases in the number of consumers of health-related foods and the amount of money spent. In particular, about $40 \%$ of Foods with Functional Claims were supplements. This suggests that supplements are central among functional foods. Many foods called supplements are provided in drink-type forms or tablet or capsule forms like medicine [3]. In a study of people aged 40 to 82 years old in Japan, it was reported that $55 \%$ of men and $61 \%$ of women used supplements [4]. From this report it is thought that the use of supplements is common among middle-aged and elderly people in Japan. On the other hand, supplements are recognized to have adverse events related to health damage, and there is concern about the safety of health-related foods [12]. Many adverse events occur with the use of products that target weight reduction in young women. These kinds of health damage have also been reported in dieting [13], but the use of diet foods centered on supplements has been steadily increasing. It may be that there is much health damage in middle-aged and elderly people from dieting. This can be said because data related to diet effects have not investigated the effects of aging. In this study, the diet effect data for variation with age in the weight changes were investigated from diet information from about 70,000 cases. In the mean for all ages, a weight loss effect of about $1 \mathrm{~kg}$ was seen, but of course there were significant differences. In other words, the amount of weight loss was clarified as the diet effect. Then, to investigate the changes in weight with consideration of aging, the changes with age in the amount of change in body weight were analyzed by applying the WIM to the amount of change in body weight for each age cohort. Gradually decreasing trends were seen in the amount of change and the rate of change with age in body weight and the change rate, and those curves showed gradual decreases from the $20 \mathrm{~s}$ to $60 \mathrm{~s}$. As hypothesized, the decrease with age showed a gradual decline. In other words, the diet effect differs with age, and if older people expect the same kind of effect as younger people, there is a possibility that conversely health damage will be induced. In this study, we investigated the age at which clear differences appear in the diet effects, including health damage. The age-related changes in the diet effect can be described with the WIM, and by differentiating the age-related distance curve the age-related inflection point in the diet effect was identified from the velocity change. That inflection point was at 30 years old. The diet effect enters a decreasing phase where the effect decreases at a critical age of 30 years old. It may be that differences in the diet effect are seen due to variations in 
metabolic mechanisms with age. Basal metabolism is deeply involved in those metabolic mechanisms.

It is reported that basal metabolism generally accounts for $60-75 \%$ of daily energy consumption, and is affected by daily activity, food intake, and lean body mass $[14,15]$. That means that basal metabolism accounts for $60-70 \%$ of total daily calories burned, while the remaining approximately 30 of calories are consumed in physical activity including exercise. Therefore, even with exercise, weight loss cannot be achieved easily without raising basal metabolism. [16] Indicated that at rest the amount of energy consumed by muscles is about $22 \%$ of basal metabolism, which suggests that the amount of energy consumed in exercise really is low. Therefore, while easy diet foods have appeared, basal metabolism clearly cannot be ignored. It has been reported that the basal metabolism of young women today is lower than that of women in the 1960s [17]. It has also been suggested that in women basal metabolism declines from around the age of 30-40 years old [16]. Thus, basal metabolism has a huge effect in dieting, and even if a person takes diet supplements the results will differ depending on individual differences in basal metabolism. Differences in diet effects are seen with age, as in the findings in this study. Moreover, a critical phase of 30 years old was shown for the diet effect. In the phenomenological aspect of the actual diet effect, however, the behavior of the velocity curve becomes gentler at about 40 years old. Therefore, around 40 years old is thought to be a physical transition phase in women, with 30 years old as a critical point. These findings indicate that the age-related changes in basal metabolism may be greatly involved. It may also be considered that the age of 30 may is a critical phase for basal metabolism and that the age of 40 is the transition phase for that basal metabolism. This may suggest a physical transition phase specific to women.

\section{Conclusion}

The etymology of the word "diet" derives from the Greek "dieta" (lifestyle, way of living), and from the late 19th century a general trend arose in Western countries for corpulence to be viewed as something that is not good. In recent years the word diet has become established as an abbreviation for "diet control" or "diet therapy" [1]. Dieting has become an inseparable behavior for many women, and behaviors that depend on simple diet supplements are an inevitable phenomenon. In this study, diet effects were seen from data for about 70,000 cases in women, but the fact that differences were shown in the diet effect with age is a noteworthy finding. The WIM was applied to the age-related changes in the size of the diet effect, and the identification of the age of 30 as a critical point in the diet effect from the behavior of the velocity curve may be considered a finding derived for the first time. The slowing of the velocity then becomes consistent about 10 years after the critical point, at around the age of 40 , from which it may be proposed that the age of 40 is a true transition phase for women.

\section{References}

[1] Unno. H, "History of diet - corset not seen," Shinshokan, Tokyo. 1998. (in Japanese).

[2] Stearns. P. N, "Fat History: Bodies and Beauty in the Modern West,” NYU Press, 2002.

[3] Sharpe. P. A, Granner. M. L, Conway J. M, Ainsworth. B. E, and Dobre. M, "Availability of weight-loss supplements: Results of an audit of retail outlets in a southeastern city," J Am Diet Assoc, Vol. 106, pp. 2045-2051. 2006.

[4] Imai. T, Nakamura. M, Ando. F, and Shimokata. H, "Dietary supplement use by community-living population in Japan: data from the National Institute for Longevity Sciences Longitudinal Study of Aging (NILS-LSA)," J Epidemiol, 16 (6), pp. 249-260, 2006.

[5] Lenz. T. L, and Hamilton. W. R, "Supplemental products used for weight loss," J Am Pharm Assoc, Vol. 44, pp. 59-67. 2003

[6] Bailey. R. L, Gahche. J. J, Miller. P. E, Thomas. P. R, and Dwyer. J. T, "Why US adults use dietary supplements," JAMA Intern Med, Vol. 173, pp. 355-61. 2013.

[7] Blanck. H. M, Serdula. M. K, Gillespie. C, Galuska. D. A, Sharpe, P. A, and Conway. J. M, et al. "Use of nonprescription dietary supplements for weight loss is common among Americans.” J Am Diet Assoc, Vol. 107, pp. 441-7, 2007.

[8] Fujii. K, and Yamamoto. Y, "The analysis of the growth velocity curve in height based upon the maturity rate," Japan Journal of Physical Fitness and Sports Medicine, Vol. 44, pp. 431-438, 1996.

[9] Fujii. K, and Matsuura. Y, "Analysis of velocity curve derived from mean growth curve in physique of boys," Japan Journal of Physical Education, Health and Sport Science, Vol. 41, pp. 247-260, 1996.

[10] Fujii. K, and Matsuura. Y, "Analysis of the growth velocity curve for height by the Wavelet Interpolation Method in children classified by maturity rate," American Journal of Human Biology, Vol. 11, pp. 13-30, 1999.

[11] Fujii. K, "Scientific approach to growth and development -Physical information science of growth and development and health-," Sankeisha, 2006. (in Japanese).

[12] Japan Food Newspaper, "Health-related food special feature," https://news.nissyoku.co.jp/news/muraoka2020051107133707 4, 2020. (in Japanese).

[13] Kobayashi. E, Sato. Y, Umegaki. K, and Chiba. T, “Analysis of Safety Alerts Associated with Dietary Supplements from Japan and Overseas," Shokuhin Eiseigaku Zasshi, Vol, 59 (2), pp. 93-98, 2018.

[14] Goto. J, Konno. H. Shida. J, Sugawara. K, Uchida. K. and Shibata. F, "Relationship between resting Oxygen uptake and Grip Strength," Yamagata Journal of Health Sciences, Vol. 18, pp. 31-36. 2015.

[15] Snitker. S., and Macdonald. I, "Ravussin E and Astrup. A. "The sympathetic nervous system and obesity: role in aetiology and treatment," Obes Rev 1, pp. 5-15, 2000.

[16] Tanaka. K and Nakata. Y, "It is possible to increase muscle mass and basal metabolic rate during weight loss?" Jpn J Phys Fitness Sports Med, Vol. 66 (3), pp. 209-212, 2017. 
Katsunori Fujii et al: $\quad$ Examination Regarding Change of Differences in the Diet Effect with Age: Analysis Based on Wavelet Interpolation Model

[17] Takahashi. E, Higuchi. M, Hosokawa. Y and Tabata. I, "Basal Metabolic Rate and Body Composition of Japanese Young Adult Females,” Jpn. J. Nutr. Diet, Vol. 65 (5), pp. 241-247, 2007. 